\title{
Tweet, Truth and Fake News: A Study of BJP's Official Tweeter Handle
}

\author{
Dr.Amit Sharma ${ }^{1}$ and Ms.Aayushi Goyal ${ }^{2}$
}

\begin{abstract}
Twitter is one of the strongest media for communication among the certain public. Unfortunately, such a beautiful mass communication medium is now used for spreading rumours, fake news and false information. The present study is an attempt to understand the agenda, propaganda, media priming and media interpretation of BJP's official tweets. The objectives of the study are to know media priming, to know truthfulness and to know media interpretation of BJP's official tweets. The content analysis method is applied to data collection. The result indicates that most of the tweets posted on BJP's official Twitter handle have micro or positive agenda and no any propaganda. Tweets which are used in news as un-cocked information presented very neutral and positive way. These interpretations clearly show that tweets from BJP's Twitter handles are not primed by Media. It means tweets having popular faces, controversial statements, fake information are primed by mass media.
\end{abstract}

Key words: Twitter, Political Communication, Agenda \&Propaganda in tweets, Media Priming, and Media interpretation.

\section{Introduction:}

The key responsibility of the mass media is to disseminate positivity in the society. Mass communication has the feature of spreading any information in a short moment. For this, the social media platform Twitter is the strongest medium. Unfortunately, such a beautiful mass medium is now used as a tool for spreading rumors.

A well-known proverb "a lie can travel the whole world while true will be put in shoes" clarify the power of lie and weakness of truth. Twitter is also suffering from the same situation. Through a fake tweet's, un-traceability any reality got killed. A hero can be a villain within hours. The Twitter team is also aware of this. Just a few fake and conspiracy based tweets dominate during elections. Therefore, they are trying to resolve this issue. This research paper deals with these all aspects. For any political party, fake news creates more problem. Keeping in mind this the researcher has chosen BJP twitter handle.

\section{Twitter; a powerful communication tool}

Twitter is known as a social networking platform which disseminates various type of information. It is used to circulate facts, information, idea or thought that is known as a tweet. Earlier, the tweet was restricted in 140 characters. But, today the maximum character in the tweet is expended up to 280 in most languages. In 'The age of Twitter: Donald J. Trump and the politics of debasement', Ott (2017) claimed that after the arisen of Twitter, the discourse of society has been

1 Assistant Professor, Department of Journalism and Mass Communication, JECRC University, Jaipur

2 Student, Department of Journalism and Mass Communication, JECRC University, Jaipur changed. Through Twitter, public discourse becomes very easy but thoughtless and unmannered.

One of the limitations of twitter is that only registered users can tweet and re-tweet. But registration is very easy and possible through the website or mobile app. In their work 'Fake news detection on social media: A data mining perspective', Shu, Sliva, Wang, Tang, \&Liu(2017) posited that social media for news consumption is a double-edged sword. It is low budgeted, smooth access, everywhere available medium which have the capability of fastest dissemination of information. Although, it can be used for fake news and misinterpretation.

Oxford Dictionary defines the tweet as 'a post on the social media application/site Twitter'. Urban Dictionary explains tweet as a post on Twitter.Wiktionary.org explained the tweet as an entry posted on the micro-blogging service i.e. Twitter. Twitter is a powerful communication tool. Anyone can imagine the popularity twitter with the facts below. In 2012, 100 million users are using Twitter globally. They tweet average 340 million tweets per day. In 2013, Twitter awarded as a most visited website. Now, Twitter becomes a major tool of information, agenda setting, and propagation of political ideas. During the 2016 American election campaign, Twitter circulates the highest news related to politics. According to Eastern Times report, about 40 million elections related tweet sent by people on American pool day. It was a record for news and twitter history. If we took sight on Indian general election 2014, social media played a very strong role in public opinion making and changing their voting behaviour. In 'Hoaxy: A platform for tracking online misinformation' Shao, Ciampaglia, Flammini, \&Menczer (2016) posited that there is a 
new trend in social media to spread misinformation, rumours, and fake news. Several institutions are using this platform to meet their propaganda and agenda setting. Few scholars claimed that social media is useful as well as harmful. In 'Defining "fake news" A typology of scholarly definitions', Tandoc, Lim \& Ling (2018) suggested that fake news is basically a mixture of fabrication, manipulation, propaganda and news satire.In their study 'The Science of Fake News', Laser et al. (2018) posited that the problem of fake news is global. This is not only related to Journalism and Mass Communication stream but Computer Science stream facing the same problem.

\section{Political Communication and Social Media}

Political communication refers to information disseminated within the public that can influence politics and policy makers along with mass media and citizens. Generally, information circulates over mass medium but now social media platforms are also a popular tool of information distribution. Blumler\& Kavanagh (1999)claimed that mass media can persuade society and can shape political communication in the democratic system. Another scholar Enli \& Skogerbo (2013) posited that social media generally focuses on an individual politician. With the help of social media, the political player tries to convince the public by focusing on issues related to their interest. Social media is widely used for personalized campaigning.

Opinion leaders and the political player also use social media for dissemination of their ideology and perception on certain issues. Gokce, Hatipoglu, Gokturk, Luetgert, \&Saygin (2014) posited that social media has the power to shape political attitude. This media platform provides space for political socialization and mobilization. Opinion leaders use Twitter to motivate their followers and political engagement. This microblogging service is used to mobilization and public expression. Opinion maker uses Twitter to contribute in the political communication process. Wilson (2011) posited that politics is mediatized by social media. Twitter is used to inform about daily activity.

\section{Importance of Study}

Information is power. Today, no one can survive in society without information. People can get information from mainstream media and social media. The present study deals with social media i.e. Twitter and use of tweet by various mainstream media. The importance of the present study is as follows
1. This study is very helpful to understand the agenda and propaganda in political tweets.

2. The presentstudy is useful to understand the presentation or use of tweet in news as a source of information.

3. With the present study, the researcher can understand the relationship between a political tweet and media priming.

4. This study is also useful to understand the truthfulness of political tweet in mass media.

5. The present study is useful to understand the media interpretation of political tweets as truth or fake news.

\section{Review of literature}

This part of the present study contains a review of the existing literature available on Twitter, political communication, fake news and misinformation. The studies have been presented in chronological order so that the oldest studies are presented first followed by the latest. Review of literature consists of the theoretic and practical framework.

In a study, 'Journalists, Social Media, and the use of Humor on Twitter', Holton \& Lewis (2011) examine the use of social media platforms by the mainstream media's journalists. The researcher conducted a content analysis of about 22 thousand tweets.The result indicate that a journalist's use of humor is directly associated with sharing a viewpoint and personal life details and engaging in interpersonal discourse. In another study 'Faking sandy: characterizing and identifying fake images on twitter during hurricane sandy', Gupta, Kumaraguru, \& Joshi (2013) examined the role of Twitter, during Hurricane Sandy (2012) to extent fake pictures about the disaster. The researcher analysed 10,350 unique tweets which were circulated on Twitter, during this period. It is found that 86 percent of tweets spreading the fake images were retweets.

In a study 'Epidemiological modeling of news and rumours on twitter', Jin, Dougherty, Saraf, Cao, \& Ramakrishnan, (2013) analysed the information diffusion on social media platforms like Twitter. The researcher used epidemiological models to describe information flows in twitter resulting from both news and rumours. The result indicates that Twitter has become a venue to broadcast rumours and misinformation. In another study 'Twitter as a reporting tool for breaking news: Journalists tweeting the 2011 UK riots', Vis (2013) studied the two journalist's activity on Twitter. The researcher found that both journalists tweeted continuous four day's during 2011 UK riots and they used Twitter as a tool of reporting. It proved that Twitter is also 
useful for conventions, the taking and sharing of images and the sharing of mainstream media content.

In a study 'Fake news: The narrative battle over the Ukrainian conflict' Khaldarova \& Pantti (2016) examined the use of the internet as a tool of information warrior against distorted information and fake news. The result indicates that social media platform is able to fight against any propagated narrative and misinformation. Twitter users judged the accuracy of these news stories and contributed to the establishment or extinguish of the agenda. In a research work 'Fake news or truth? using satirical cues to detect potentially misleading news', Rubin, Conroy, Chen, \& Cornwell (2016) analysed the satire is an interesting subject for news writing. Researcher given a conceptual overview that elaborating, and illustrating are the unique features of satirical news. It copied the format and style of journalistic reporting.'

In a study 'Social media and fake news in the 2016 election', Allcot t\& Gentzkow (2017) studied the false stories or fake news spread through social media. After content analysis, the researcher found that social media is an important source of information but not the dominant source of election news. People like to believe such stories that favour their favourite candidate, especially if they have ideologically separated social media platform. In a research work 'Fake news detection on social media: A data mining perspective', Shu, Sliva, Wang, Tang, \& Liu (2017) analyzed the social media use and its impact on society. The researcher used survey method to meet the result. The result indicate that it is very challenging to use social media for news consumption. Its low cost, easy access, and quick circulating of information quality lead people to consume news from social media. But, social media enables the easily spread of fake news or false information. The dissemination of fake news puts negative impacts on individuals and society.

In a research work 'Junk news and bots during the US election: What were Michigan voters sharing over Twitter', Howard, Bolsover, Kollanyi, Bradshaw, \& Neudert (2017) examined the dissemination of propaganda over social media platforms. Researchers collected data on automation and junk news using main hash tags related to politics in Michigan state during the 2016 US Presidential Election. The resultindicates that the grouping of automation and propaganda is significantly affected public opinion during significant policy debates, elections, and political disasters.
In a research work, 'The fake news spreading plague: was it preventable?', Mustafaraj \& Metaxas (2017) examined how social networks are used to spread misinformation. They studied the organized effort to circulate misinformation about Martha Coakley through anonymous Twitter accounts. Researchers find out that a community of users use this fake information and extend it to other social networking platforms. In a study 'Examining the Alternative Media Ecosystem Through the Production of Alternative Narratives of Mass Shooting Events on Twitter', Starbird (2017) examined the Twitter and alternate mass media. The researcher collected tweets related to alternative narratives like conspiracy theories. The result indicates that alternative news sites propagate and shape alternative narratives, while mainstream media deny this kind of narratives.

Research Methodology:This part contains objectives,hypothesis, and research design.

Objectives of the Study: The objectives of the research are given below.

RO1: To know the agenda in BJP's Official tweets.

RO2: To know the propaganda in in BJP's Official tweets.

RO3: To measure the media priming in $\mathrm{BJP}^{\prime} \mathrm{s}$ Official tweets.

RO4: To know the truthfulness of BJP's Official tweets.

RO5: To know the media interpretation of $\mathrm{BJP}^{\prime} \mathrm{s}$ Official tweets.

Hypothesis of the Study: The hypothesis of the research is given below.

Ha1.There is an association between the agenda in tweets and media priming.

Ha2.There is an association between the propaganda in tweets and media interpretation.

\section{Research Design}

The research design of the present study is quantitative cum qualitative in nature, as the study aims to analyse the tweet on the qualitative parameter.

Variables: In the present study, both dependent and independent variables are used to meet the objective. Agenda, Propaganda, Media Priming and Media Interpretation are the few important variables.

Sample Design: The sample design of the present study comprises the following elements: 
A. Universe-All the tweets of Indian National political party'sofficial Twitter handle constitute the universe of the study.

B. Sampling Element: It is very difficult to examine the whole population,the Official Twitter handle of BJP (largest party of the world) has been selected as the sampling frame for the present study.

C. Sampling Unit: Sampling unit of present study is the tweets of the official Twitter handle of BJP i.e. https:/ / twitter.com/bjp4india

D. Sampling Technique: Systematic sampling method has beenfollowing to collect the data from the official Twitter handle of BJPduring theselected time period.

\section{Period of study:}

Period of the present study is two months. The researcher has collected tweets posted on BJP's official Twitter from June 2018 to July 2018. Data has been collected after 3 days of a tweet posted, because,within three days, the tweets have got highest popularity, retweet and like or dislike.

\section{Nature and Sources of Data}

In the present study, only primary data has been used as a requirement of the research objectives. The primary data has been collected through content analysis. The codebook is prepared on the basis of priori coding.

Analysis: Analysis of the study is divided into two part. Part first consist of percentage analysis and part second consist of chi-squire test for hypothesis testing.

Agenda in BJP's Official tweets

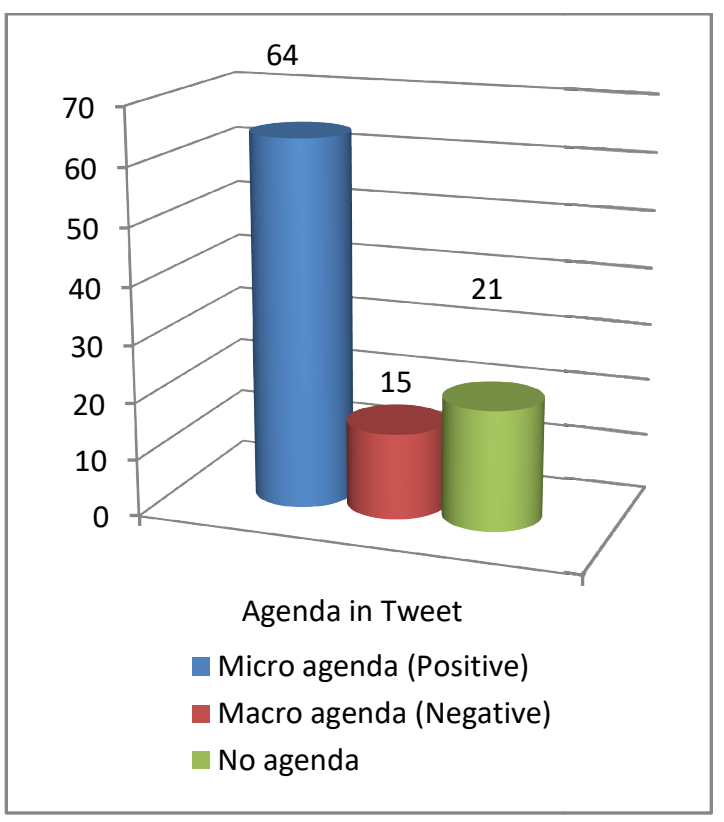

Each person has a viewpoint or ideology. This kind of ideology reflects from his/her tweet. Few tweetsis posited for a special purpose. This purpose is known as an agenda. Media coverage is also linked to the attainment of the rise of a politicalissue. Mass Media is also havingan effect on the political agenda.After analyzing tweeter of BJP'sofficial Twitter account,it is found that $64 \%$ tweets have micro agenda or positive ideology. It means about two third tweets have something positive or productive ideology. Another 21\% tweets have no agenda. However, the rest $15 \%$ tweets have micro agenda or negative ideology.

Measurement of Propaganda in BJP's Official tweets

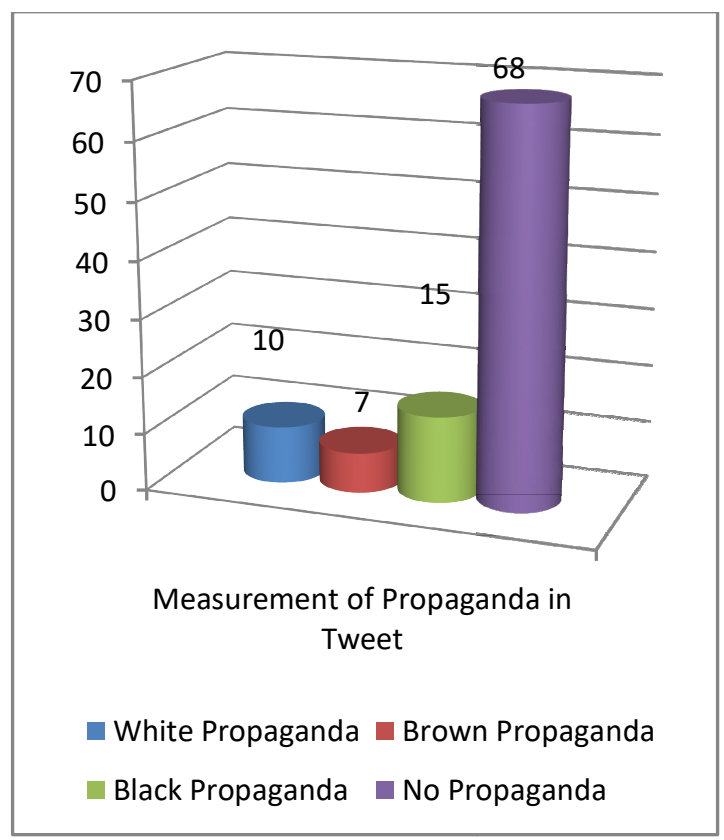

Propaganda is a kind of information which is supposed as inaccurate. The political organization publishes or broadcasts this kind of information in order to influence people. Actually, propaganda's nature is never objective. After analyzing the Tweeter of BJP's official Twitter account, it is found that $68 \%$ of all tweets have no propaganda. It means they are disseminating actual information without any manipulation in fact. Another $10 \%$ of total tweets have white propaganda, it means these tweets have accurate fact but presented in propagated manner. $15 \%$ of total tweets have black propaganda; it indicates that these tweets have inaccurate information. Rest $07 \%$ of total tweets has brown propaganda; it means tweets have fact, but accuracy is uncertain. 
Media Prime Political Tweet as News

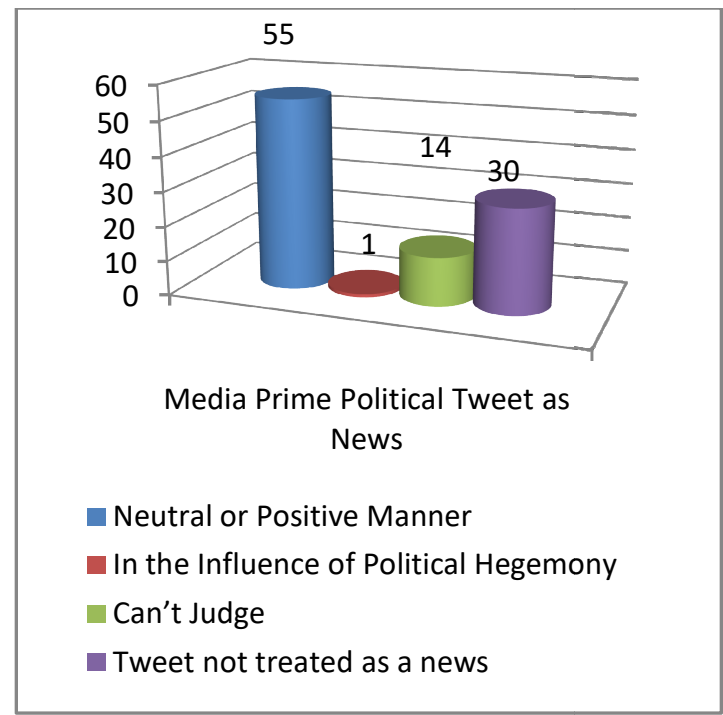

Priming is very common in political communication. Media house got various type of information from many sources. After that, this information was published/broadcast on the basis of specific agenda or purpose. Only a few issues are raised and rest information sent to dustbin or recycle bin, this is a kind of priming the news. After analyzing BJP Official Twitter account, it is found that $55 \%$ of total tweets are presented in news as neutral or positive manner. Only $1 \%$ of total tweets are presented in news in the influence of political hegemony. Another 30\% of total tweets do not becomepart of the news.Rest $14 \%$ of total tweets become part of the news. But, these tweets are presented such a way that can't judge as neutral/positive or influenced by political hegemony.

Media prime the tweet by focusing on some issues more than others.

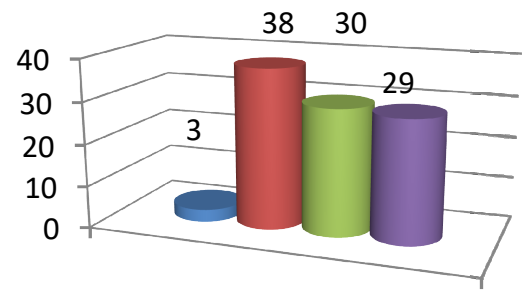

Media Prime the Tweet. (By focusing on some issues more than others)

Media focus on the tweet more than other news

Media focus on the tweet average

Media focus on the tweet below the average.

Media not focus on tweet
Priming is a very popular phenomenon in media to keep benefitted their desired political party. For this the concern,media house focus on the specific issue to change public perception.After analyzing BJP Official Twitter account,it is found that $38 \%$ of total tweets got average focusby mass media. It means thatmedia did not give more importance to these tweets more than another issue.Another $30 \%$ of total tweets got below the average focus bymedia.It means that the media gave very less importance to these tweets more than other issues.Another 29 percent of total tweets did not get focusby media;it indicates that these tweetsnot having newsworthiness.Only three percent of total tweets were primed by mass media.It means that the media gave more importance to these tweets more than others issues.

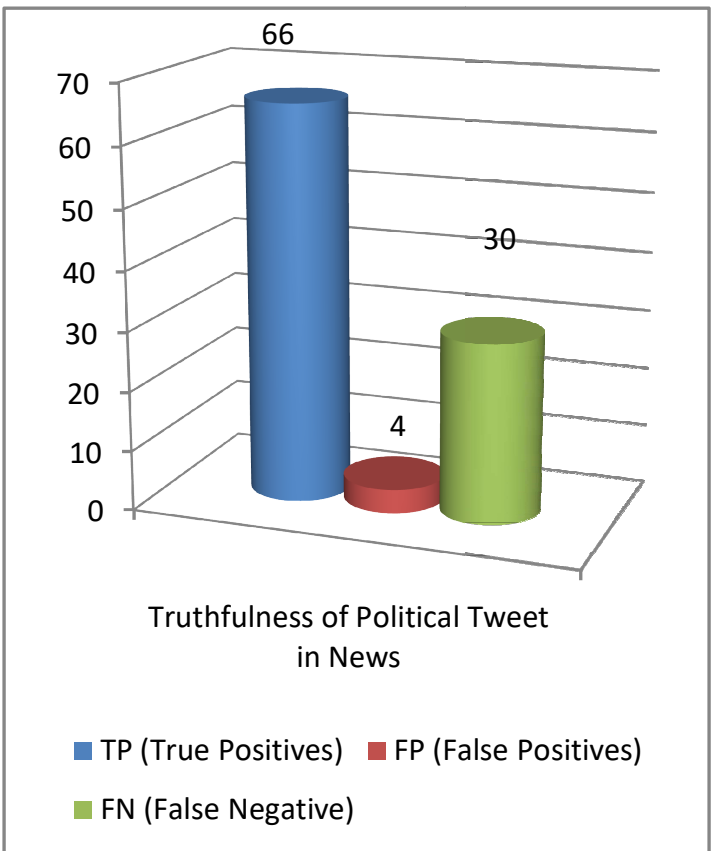

Truthfulness of BJP's Official tweetsin News

Bewith the truth is the key responsibility of media.For this,Media attempts to find the truth which is missing.After analyzing the newswhich isbased on BJP official tweets, it is found that $66 \%$ of total tweets are presented in news astrue positive manner.It means that tweets which are used in news are correct.Only $04 \%$ of total tweets are presented in news as false positive manner. It means that tweets which are used in news are incorrect.Rest $30 \%$ of total tweets are not presented in news. It is indicated as a false negative. 


\subsection{Analysis of Media Interpretation of BJP's Official tweets}

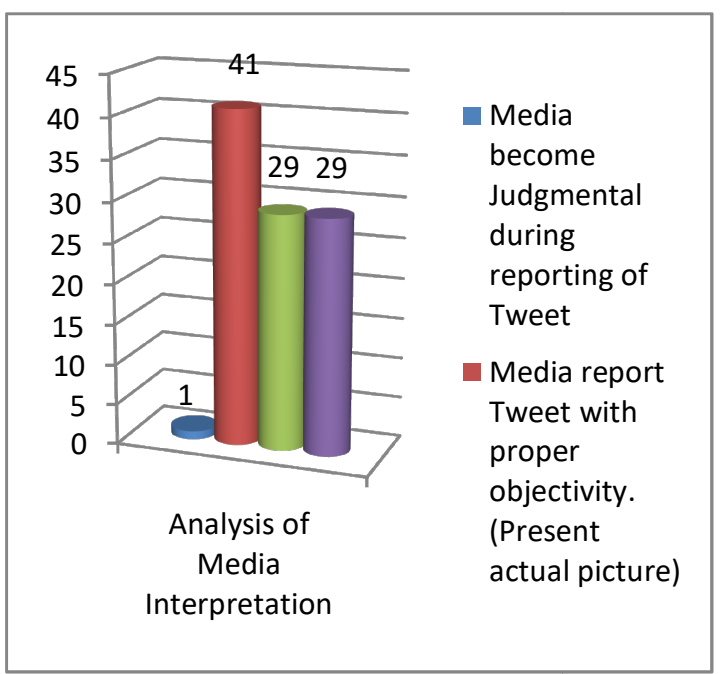

Interpretation is a communication process.The journalist got information from the various source and prepare news after interpretation of that information. Hence, media interpretation is not simply presenting information, but to translate media message in a strategic way.After analyzing the news which is based on BJP official tweets, it is found that media report $41 \%$ of total tweets with proper objectivity. It means tweets presented in an actual way. Media reported another 29\% of total tweets without ethical interpretation. It means tweets missed its objectivity. On the other hand, $29 \%$ of total tweets not become part of any news. It means these tweets are not having media material. Only $1 \%$ of total tweets reported by media with negative interpretation.It means media become judgmental during reporting of these tweets.

\section{Test of Significance}

Ho. There is no any association between the agenda in tweets and media priming.

\begin{tabular}{|l|l|l|}
\hline \multicolumn{3}{|c|}{ Chi-Square Test } \\
\hline Pearson Chi-Square & $\mathrm{df}$ & Asymp. Sig \\
\hline 9.702a & 6 & .138 \\
\hline
\end{tabular}

There is no significant relationship between the agenda in tweets and media priming of tweets because $\mathrm{p}$-value $(\mathrm{P}-0.138)$ is more than significance level 0.05 . So, the null hypothesis 'there is no any association between the agenda in tweets and media priming' is failed to reject.

\subsection{Test of Significance}

Ho.There is no any association between the propaganda in tweets and media interpretation.

\begin{tabular}{|l|l|l|}
\hline \multicolumn{3}{|c|}{ Chi-Square Test } \\
\hline Pearson Chi-Square & df & Asymp. Sig \\
\hline $17.008 a$ & 09 & .049 \\
\hline
\end{tabular}

There is asignificant relationship between the propaganda and media interpretation of tweets because p-value (P-0.049) is less than significance level 0.05 . So, the null hypothesis 'There is no any association between the propaganda in tweets and media interpretation' is rejected.

\section{Results}

1. Most of the tweets posted on BJP's Official Twitter handle have micro or positive agenda and on any kind of propaganda.Tweets which are used in the news as information has presented very neutral and positive manner. These interpretations clearly showthat tweets from BJP's Twitter handles are not primed by Media.It means tweets having popular faces,controversial statements,fake news and fake information are primed by mass media.

2. Tweets of BJP's Official Twitter account are primed in news but most of them are in a neutral or positive manner.Very few tweets are primed in news with the influence of political hegemony.

3. Truth is the base of all news. News which are developed from tweets is based on truth. Very few such news published which have present tweets as distorted manner.

4. Media report BJP's official tweets with proper objectivity.Tweets are used in news as a source of information in an accurate manner. Although few tweets as used as the distorted manner and presented as fake news.

5. Agenda in tweets and tweets primed by mass media not associated with each other. But, there is an association between the propaganda in tweets and its media interpretation.

\section{References}

\section{Journal}

Allcott, H., \&Gentzkow, M. (2017).Social media and fake news in the 2016 election. Journal of Economic Perspectives, 31(2), 211-36.

Holton, A. E., \& Lewis, S. C. (2011).Journalists, social media, and the use of humor on Twitter. Electronic Journal of Communication, 21(1/2).

Howard, P. N., Bolsover, G., Kollanyi, B., Bradshaw, S., \&Neudert, L. M. (2017). Junk news and bots during the US election: What were Michigan voters sharing over Twitter. Computational Propaganda Research Project, Oxford Internet Institute, Data Memo, (2017.1). 
Khaldarova, I., \&Pantti, M. (2016). Fake news: The narrative battle over the Ukrainian conflict. Journalism Practice, 10(7), 891-901.

Lazer, D. M., Baum, M. A., Benkler, Y., Berinsky, A. J., Greenhill, K. M., Menczer, F., ...\&Schudson, M. (2018). The science of fake news. Science, 359(6380), 1094-1096.

Ott, B. L. (2017). The age of Twitter: Donald J. Trump and the politics of debasement. Critical Studies in Media Communication, 34(1), 59-68.

Shu, K., Sliva, A., Wang, S., Tang, J., \& Liu, H. (2017). Fake news detection on social media: A data mining perspective. ACM SIGKDD Explorations Newsletter, 19(1), 22-36.

Starbird, K. (2017, May). Examining the Alternative Media Ecosystem Through the Production of Alternative Narratives of Mass Shooting Events on Twitter. In ICWSM (pp. 230-239)

TandocJr, E. C., Lim, Z. W., \& Ling, R. (2018).Defining "fake news" A typology of scholarly definitions. Digital Journalism, 6(2), 137-153.

Vis, F. (2013). Twitter as a reporting tool for breaking news: Journalists tweeting the 2011 UK riots. Digital journalism, 1(1), 27-47.

\section{Conference Proceeding}

Gupta, A., Kumaraguru, P., Castillo, C., \& Meier, P. (2014, November).Tweetcred: Real-time credibility assessment of content on twitter. In International Conference on Social Informatics (pp. 228-243).Springer, Cham.
Jin, F., Dougherty, E., Saraf, P., Cao, Y., \&Ramakrishnan, N. (2013, August).Epidemiological modeling of news and rumours on twitter.In Proceedings of the 7th Workshop on Social Network Mining and Analysis (p. 8).ACM.

Rubin, V., Conroy, N., Chen, Y., \& Cornwell, S. (2016).Fake news or truth? using satirical cues to detect potentially misleading news. In Proceedings of the Second Workshop on Computational Approaches to Deception Detection (pp. 7-17).

Shao, C., Ciampaglia, G. L., Flammini, A., \&Menczer, F. (2016, April).Hoaxy: A platform for tracking online misinformation. In Proceedings of the 25th international conference companion on world wide web (pp. 745-750). International World Wide Web Conferences Steering Committee.

Gupta, A., Lamba, H., Kumaraguru, P., \& Joshi, A. (2013, May).Faking sandy: characterizing and identifying fake images on twitter during hurricane sandy.In Proceedings of the 22nd international conference on World Wide Web (pp. 729-736).ACM.

Saez-Trumper, D. (2014, September). Fake tweet buster: a webtool to identify users promoting fake news ontwitter. In Proceedings of the 25th ACM conference on Hypertext and social media (pp. 316-317).ACM.

Mustafaraj, E., \& Metaxas, P. T. (2017, June). The fake news spreading plague: was it preventable?.In Proceedings of the 2017 ACM on Web Science Conference (pp. 235-239).ACM. 